\title{
Weighted near-field focusing in an array-based GPR
}

\author{
Timofey G. Savelyev, ${ }^{1}$ Alexander G. Yarovoy, ${ }^{1}$ and Leo P. Ligthart ${ }^{1}$
}

Received 29 November 2007; revised 8 April 2008; accepted 8 May 2008; published 27 June 2008.

[1] This paper presents a 3-D imaging technique for an ultra-wideband (UWB) ground penetrating radar (GPR) with a single transmit antenna and a linear receive array. The video impulse GPR working in the frequency band of $0.3-3 \mathrm{GHz}$ has been designed in IRCTR for landmine detection, i.e., for a near-field application. Installed on a vehicle it can image in one mechanical scan a strip of $84 \mathrm{~cm}$ width due to the length of array aperture. The imaging is done by software means only. The developed imaging technique combines a real aperture focusing in the array plane with a synthetic aperture focusing in the mechanical scan direction. To compensate for parasitic time delays in the array channels, a calibration procedure is also described. Owing to directional properties of transmit antenna, the distribution of signal strength over the array is nonuniform that requires an amplitude correction when focusing the real aperture. The authors analyzed how this affects the footprint of the focused array, its cross-range resolution capability and the image quality of antipersonnel plastic landmines which were buried under different array channels. The analysis bases on experimental data sets acquired in the facilities of IRCTR and TNO-DSS. As a result, the authors propose a weighted array focusing that improves the cross-range resolution and provides proper imaging of typical buried landmines.

Citation: Savelyev, T. G., A. G. Yarovoy, and L. P. Ligthart (2008), Weighted near-field focusing in an array-based GPR, Radio Sci., 43, RS4S11, doi:10.1029/2007RS003791.

\section{Introduction}

[2] The UWB radar technologies attract immense interest for various applications in particular due to a high down-range resolution and related to it imaging and classification capabilities. The four different principles of generating and receiving an UWB signal, such as transient time domain or video impulse, pseudo-noise time domain, stepped-frequency and frequency-modulated continuous wave have been implemented successfully in recent years [Taylor, 2000; Daniels, 2004]. Development of UWB arrays which can scan the area under investigation electronically with a high cross-range resolution should be recognized as the next stage in evolution of UWB radar systems aiming to deliver a high-quality 3-D image of the scene. The challenge here deals with optimization of antenna step in the array, mutual coupling between its antenna elements, grating lobes of the array pattern and operational frequency band of the system. Unlike classical narrow-band phased arrays

\footnotetext{
${ }^{1}$ International Research Centre for Telecommunications and Radar, Delft University of Technology, Delft, Netherlands.
}

Copyright 2008 by the American Geophysical Union. 0048-6604/08/2007RS003791\$11.00 which focusing employs the phase of the carrier frequency, the UWB array focusing compensates for the difference in time of arrival between the array elements with respect to a spatial focal point. For those UWB arrays working in proximity to the target (landmine detection, medical imaging etc.), another challenge arises from a near-field effect when the array focusing depends not only on direction of arrival (DOA) but on distance to the target as well.

[3] In landmine detection, one of the most challenging UWB radar applications, the use of a vehicle-mounted array GPR allows to image a minefield strip at once that increases significantly the scanning speed. Various array systems have been developed for detection of antitank and antipersonnel landmines such as Energy Focusing GPR by Geo-Centers, NIITEK GPR by Niitek, MINEREC GPR by PipeHawk, DEMINE GPR by DEMINE EU project Consortium, Multi Sensor GPR by Satimo, Common Mid Point SAR-GPR by Tohoku University [Geo-Centers EFGPR, 2000; U.S. Humanitarian Demining R\&D Program, 2007; Chignell and Hatef, 2004; DEMINE partners, 2002; Duchesne et al., 2003; Sato et al., 2004]. It is important to mention here their common feature that is the use of multiple transmit $(\mathrm{Tx})$ and multiple receive $(\mathrm{Rx})$ antennas. The mechanical 


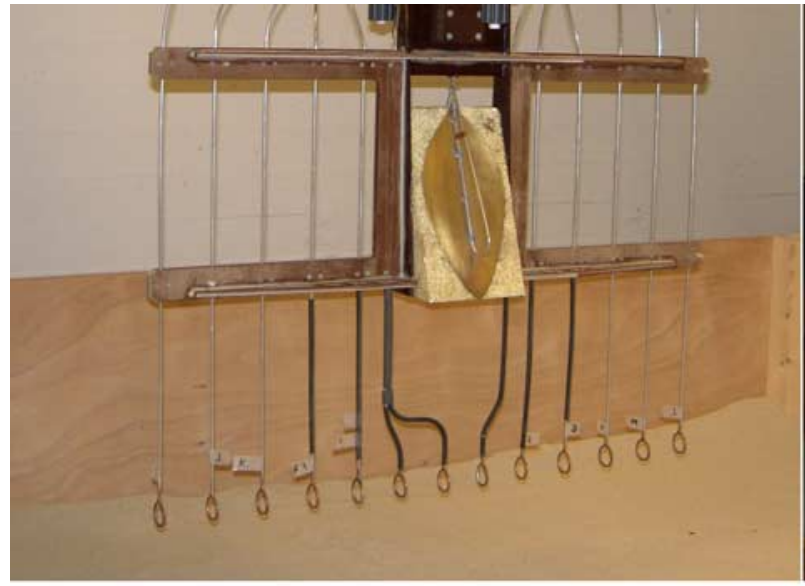

(a)

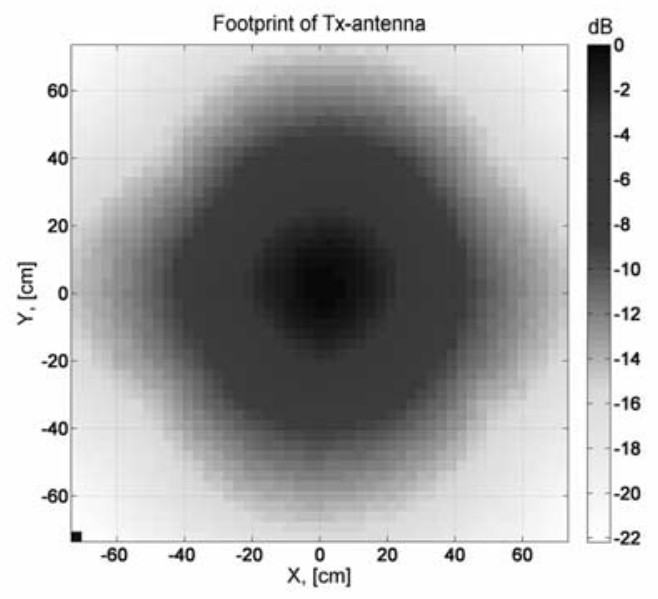

(c)

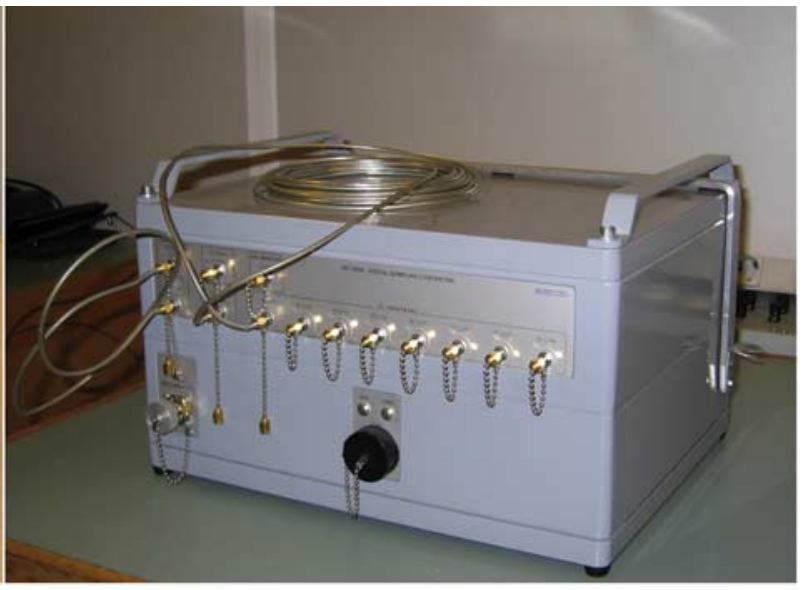

(b)

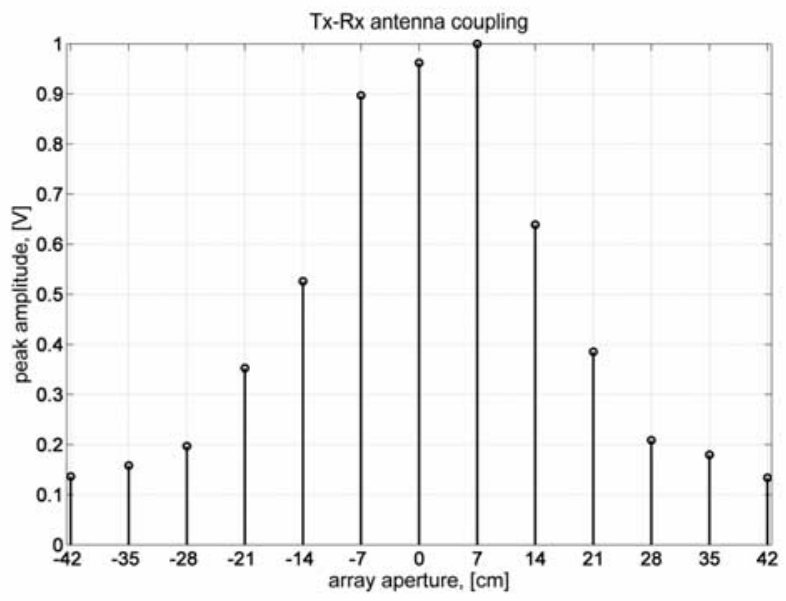

(d)

Figure 1. Array UWB radar: (a) $84 \mathrm{~cm}$ long array with a single Tx-antenna; (b) radar electronics in a waterproof case $406 \times 380 \times 260 \mathrm{~mm}$ with a weight of $14.5 \mathrm{~kg}$; (c) footprint of Tx-antenna; (d) normalized peak values of $\mathrm{Tx}-\mathrm{Rx}$ antenna coupling.

scanning in the plane of array is replaced by sequential operation of different Tx-Rx antenna pairs, which limits the speed of a carrying vehicle up to a few kilometers per hour.

[4] A new array GPR that has been designed and realized in IRCTR comprises a single Tx-antenna and a linear Rx-array with a multichannel receiver [Yarovoy et al., 2006]. Simultaneous acquisition of the array data drastically increases the scanning speed, with theoretical limit up to $148 \mathrm{~km} / \mathrm{h}$, while the whole GPR becomes more compact due to the single transmitter and specific design of the antenna system. In fact, a concept of such an improved GPR for inspection of bridges has been successfully evaluated already in nineties, without further development though [Warhus et al., 1993]. According to the concept, the vehicle with mounted GPR could have a speed of about $48 \mathrm{~km} / \mathrm{h}$ while the acquired data were processed offline. The theoretical limit of the speed is defined by the acquisition parameters, namely pulse repetition frequency, spatial sample interval, time sample interval, time window of A-scan, and number of averages, that provide appropriate quality of subsurface image. While in the minefield the carrying vehicle should move slower with processing the data online, the inspection of bridges and highways would benefit from sounding with regular speed of the vehicle and offline processing.

[5] Figure 1a shows the antenna system that includes a dielectric wedge antenna for transmission and 13 loop antennas for reception. The loops are arranged with a $7 \mathrm{~cm}$ spacing which comes from the optimization 
described in [Yarovoy et al., 2006; Zhuge et al., 2007] and results in an array aperture of $84 \mathrm{~cm}$. The electronics shown in Figure 1b is rather compact and it is realized by means of video impulse technology that provides an operational frequency band of the whole GPR from $0.3 \mathrm{GHz}$ to $3 \mathrm{GHz}$ at $-10 \mathrm{~dB}$ reference level. The footprint of Tx-antenna given in Figure 1c covers the $84 \mathrm{~cm}$ long $\mathrm{Rx}$-array approximately at $-10 \mathrm{~dB}$ for the outer loops, i.e. the amplitude distribution of the radiated field is not uniform within the array aperture. This distribution can be estimated from the peak values of Tx-Rx antenna coupling as shown in Figure 1d. Strictly speaking, the antenna coupling includes not only the footprint behavior of Tx-antenna but also the re-radiation characteristics of Rx-antennas and their mutual coupling. However, the strongest, early-time peak appearing in the Tx-Rx antenna coupling is defined mainly by the Tx-antenna footprint while the contribution of Rx-antennas comes later.

[6] The principle of 3-D imaging by such an array is to focus the acquired scattered field in each subsurface voxel by focusing the synthetic aperture in the direction of the mechanical scanning and the real aperture in the plane of the array onto this voxel. The proper imaging should account for (a) specific antenna array geometry with a single Tx-antenna, (b) nonuniform amplitude distribution of the radiated field. Both aspects are for the first time addressed in this paper and determine novelty of the approach.

[7] The paper is structured into six sections. Data preprocessing and focusing are described in section 2 . An array calibration technique is proposed in section 3 . Estimation of the footprint and cross-range resolution of the focused array is given in section 4 while section 5 presents experimental imaging results for buried antipersonnel landmines. Finally, section 6 is dedicated to discussion and conclusion.

\section{Data Preprocessing and Array Focusing}

[8] At the current stage of research the complete data processing chain is implemented offline by means of specific software developed in MATLAB ${ }^{\mathrm{TM}}$. Prior to radar imaging, a preprocessing is required by the following reasons: (1) to suppress uncorrelated thermal and quantization noise; (2) to compensate for random time drift of the electronics; (3) to remove the stationary antenna coupling and clutter that consists mainly of the spatially correlated reflection from the ground surface. The first step is done for each A-scan with a low-pass finite impulse response (FIR) filter which cut-off frequency equals to that of the sampling receiver, more specifically to $6 \mathrm{GHz}$. At the second step, the antenna coupling coming as the first, strongest received signal and followed by other signals in time is aligned in each B-scan. The third, most critical step can be implemented by combination of time gating and moving average subtraction. Time gating is effective after subtraction of antenna coupling when the reflection from the ground surface becomes the strongest. Then the noninformative part of A-scan preceding the surface reflection can be easily determined and gated (nulled). For buried targets it makes sense to gate the strongest peaks of the surface reflection as well, which can be done for every A-scan separately by simple logic of searching the strongest peak and its zero crossings. Such gating gives some robustness against the surface roughness. The remaining clutter is removed by moving average subtraction where the moving window equals to the size of diffraction hyperbola of the target. The preprocessing is performed for each receive channel of the array separately. Figure 2 illustrates its effectiveness for a small metal sphere of $2 \mathrm{~cm}$ diameter when the target signal is about 40 times lower than the antenna coupling.

[9] The array data preprocessing provides for each channel a diffraction hyperbola of the target (Figure 2b) in the mechanical scan direction that should be focused into a small "spot" or target image in the true position of the target. In GPR processing, such focusing is commonly done by migration based SAR algorithms where the most explicit one is a so-called diffraction stacking algorithm [Johansson and Mast, 1994; Groenenboom and Yarovoy, 2002; Sato et al., 2004; Gu et al., 2005; Gilmore et al., 2007]. The imaging starts with creating a regular mesh-grid of the scanned area to be imaged and then every voxel of the mesh-grid is treated by the focusing algorithm. The diffraction stacking algorithm computes the hyperbola based on signal travel times, picks up amplitudes of the received signal in the respective points of B-scan, sums them up and assigns the result to the current voxel. In other words, the points of hyperbola are being migrated into its apex. The focusing of the array aperture uses the same principle.

[10] For the sake of clarity, the same meaning is given in this paper to the terms "focusing", "migration" and "imaging". Strictly speaking, the focusing relates to synthetic or real radar aperture, the migration works with the data acquired within the aperture, and the imaging concerns a target or scanned area.

[11] The data acquisition with our array GPR can be seen in Figure 3a on example of a surface laid metal sphere while the imaging geometry for a more complicated case with buried target is shown in Figure $3 \mathrm{~b}$. The coordinate system starts in the phase centre of Txantenna while the array plane is $z 0 x$, and the array moves in $y$-direction. Then the imaging algorithm based on diffraction stacking is given by

$$
f(x, y, z)=\sum_{k=1}^{13} w_{k} \sum_{j=1}^{M} s_{k}\left(t_{j}, y_{j}\right)
$$




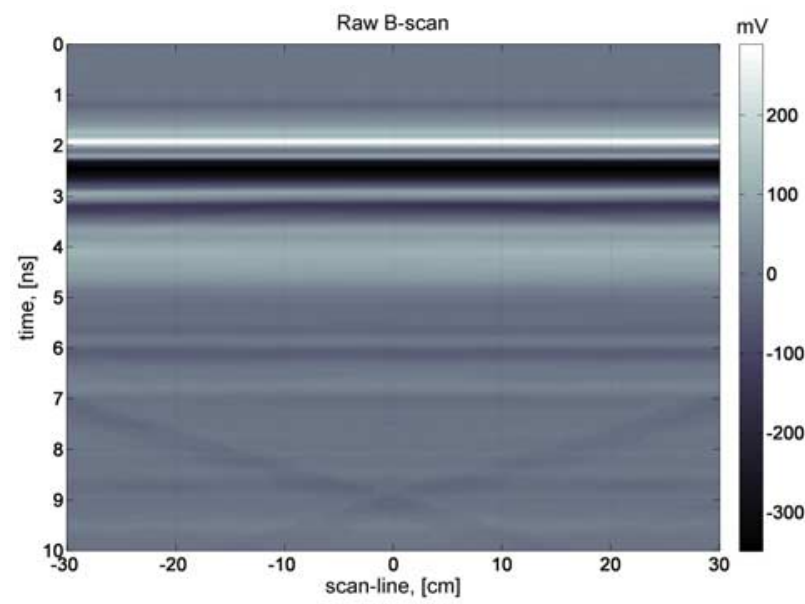

(a)

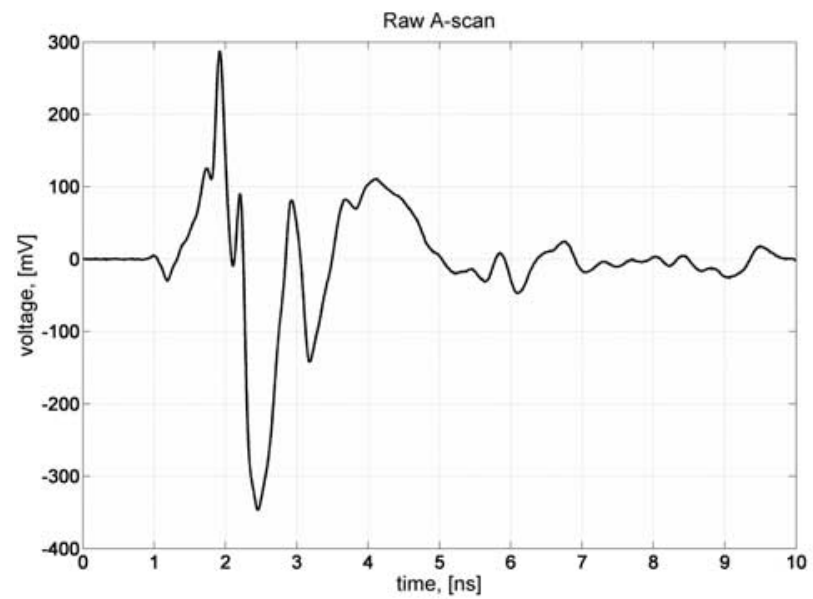

(c)

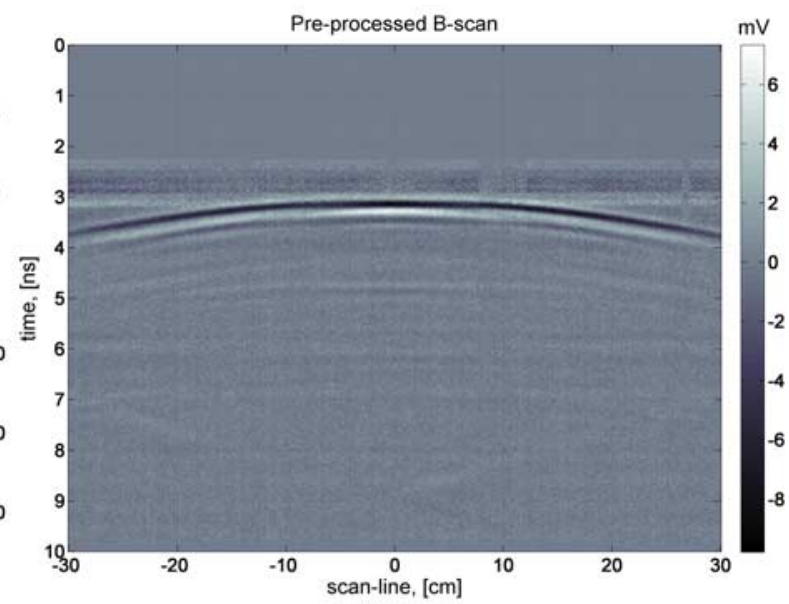

(b)

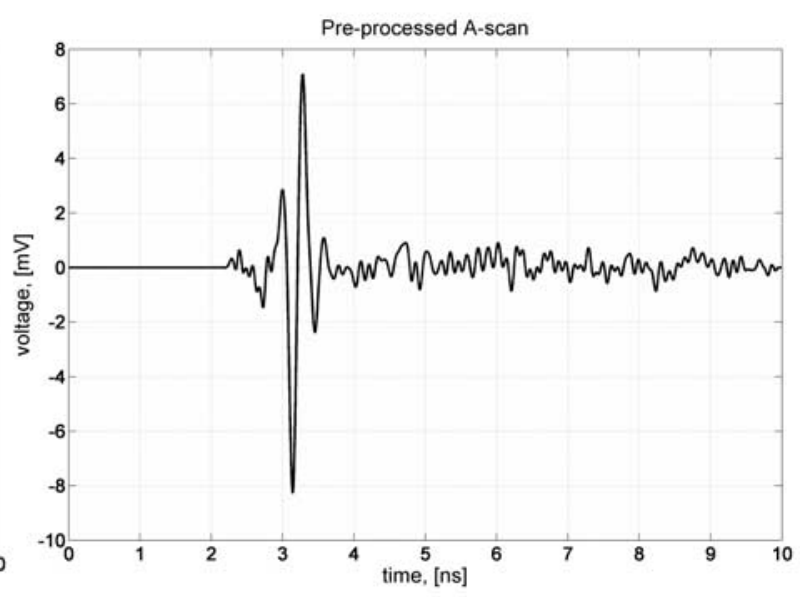

(d)

Figure 2. Data preprocessing: (a) raw B-scan acquired over a small metal sphere; (b) preprocessed B-scan; (c) A-scan taken at 0 position in the raw B-scan; (d) A-scan taken at 0 position in the preprocessed B-scan.

where $f(x, y, z)$ is a focused signal in the user-defined $L \times$ $M \times N$ mesh-grid, $w_{k}$ is a weight coefficient for the $k$-th array channel, $s_{k}\left(t_{j}, y_{j}\right)$ is a signal captured by the $k$-th antenna in position $y_{j}$ while $t_{j}$ stands for travel time for voxel $\left(x, y_{j}, z\right)$. In the conventional imaging algorithm the weight coefficients $w_{k}$ equal to 1 . The inner summation represents diffraction stacking over the line $x=y_{j}$ at depth $z$ for every array channel separately when the outer summation of the focused in $y$-direction channels is essentially diffraction stacking for the real aperture because all the respective travel times have been accounted for in the inner summation. The weights $w_{k}$ correct for a nonuniform distribution of the signal strength over the array aperture due to the Tx-antenna footprint (Figure 1c). The algorithm produces an $L \times M \times N$ image from the 13 acquired B-scans.
[12] In subsurface imaging, the signal travel time $t_{j}$ for the $k$-th array channel is defined as

$$
t_{j}=\frac{1}{c}\left(L_{j 1}+L_{j 2} \sqrt{\varepsilon}+L_{j 3} \sqrt{\varepsilon}+L_{j 4}\right)+t_{0}
$$

where $c$ is speed of light, $\varepsilon$ is relative dielectric constant of the ground, $L_{j 1}$ is distance between Tx-antenna and an input refraction point, $L_{j 2}$ is distance between this refraction point and a voxel $\left(x, y_{j}, z\right), L_{j 3}$ is distance between this voxel and the output refraction point, $L_{j 4}$ is distance between that refraction point and the $k$-th $\mathrm{Rx}$ antenna. The refraction points can be found analytically from the fourth-order equation that is derived from the imaging geometry and Snell's law. This is well described in [Gu et al., 2005]. 


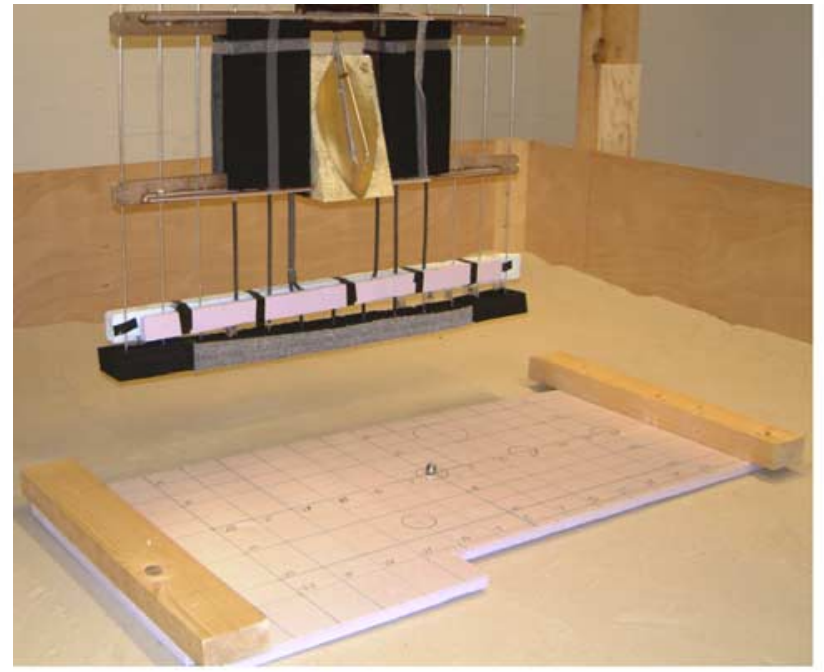

(a)

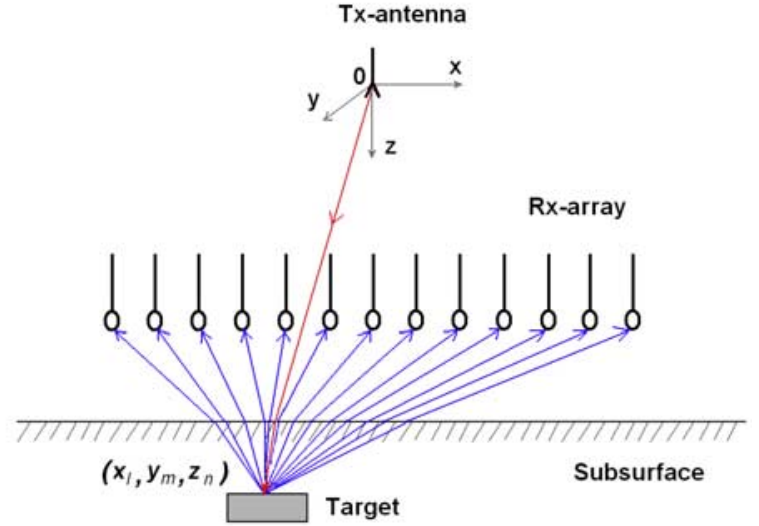

(b)

Figure 3. Array imaging: (a) scanning above a small metal sphere; (b) imaging geometry for a buried target.

[13] In addition to the signal travel time the GPR itself introduces a parasitic time delay due to propagation in the antennas, cables and receiver electronics, which should be accounted for by the summand $t_{0}$, a so-called "time zero". Therefore a calibration procedure is required to determine this parameter in every channel as well as a weight coefficient.

\section{Array Calibration}

[14] The calibration procedure allows to measure the amplitude and time correction parameters beforehand and then to use them in data processing. The amplitude correction parameters of the array channels are easy to measure as they represent the peak values of Tx-Rx antenna coupling that is a stationary characteristic of the radar. These parameters are shown in Figure 1d and their inverse values give the weights in algorithm (1).

[15] The time correction parameters can drift between switchings on and off the electronics so ideally their measurement should be done before each use of the GPR after warming up. However, their drift can be tracked in the reference channel that records a replica of the transmitted pulse, which makes possible to estimate the parameters only once and then to adjust them by comparing the reference signals. The measurement of the parameters is proposed to do with a small metal sphere ( $2 \mathrm{~cm}$ diameter in our case) placed under the middle of array as shown in Figure 3a. The radar cross section of a sphere does not depend on aspect angle while its small size makes it to be a point-like scatterer. For the given measurement geometry the SAR focusing of each channel onto plane $z 0 y$ with the sphere should give the same, correct position of the target. This can be achieved by iterative adjustment of "time zero" for every channel separately.

[16] The effectiveness of the calibration procedure is illustrated in Figure 4. A $60 \mathrm{~cm}$ long scan was made over the surface laid sphere which vertical range was $43 \mathrm{~cm}$. A voxel of the selected imaging area was chosen to be $10 \times 10 \times 3 \mathrm{~mm}$. The B-scans focused without calibration for the central and rightmost channels, as shown in Figures $4 \mathrm{a}$ and $4 \mathrm{~b}$ respectively, reveal the sphere at incorrect ranges. The properly defined "time zeros" move the sphere to the correct range in every channel (see Figures $4 \mathrm{c}$ and $4 \mathrm{~d}$ ). Furthermore, the time correction removes artifacts of the imaging. One can notice that the target image in the vertical plane consists of $2-3$ spots of different intensity which can explained by the fact that the waveform of the target signal has a few semi-periods (see Figure 2d). Also the image in the rightmost channel is somewhat blurred in comparison with that of the central channel but the reason here comes from the dependence of impulse response of UWB antenna on direction of arrival (DOA). The antenna impulse response becomes longer with larger DOA while the frequency response loses high frequencies. Another important detail regards the image intensity that is $10 \mathrm{~dB}$ lower for the edge channel than for the central 


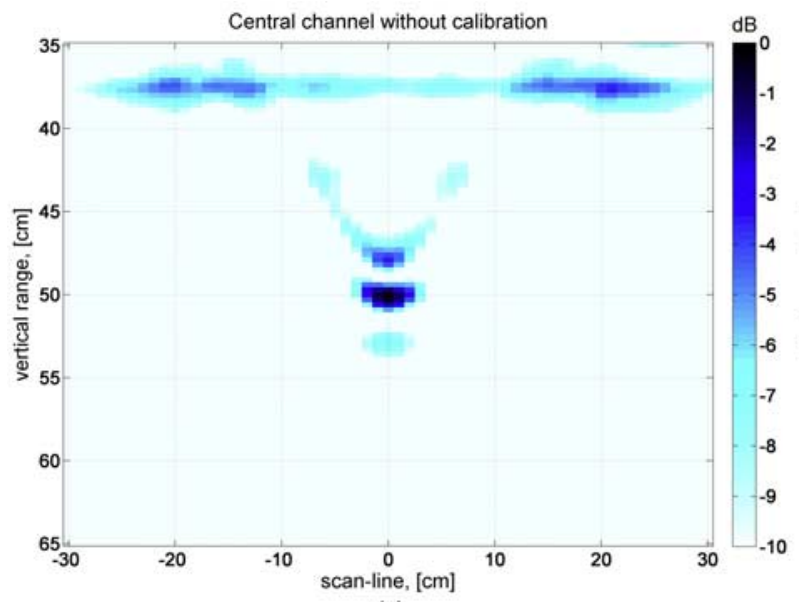

(a)

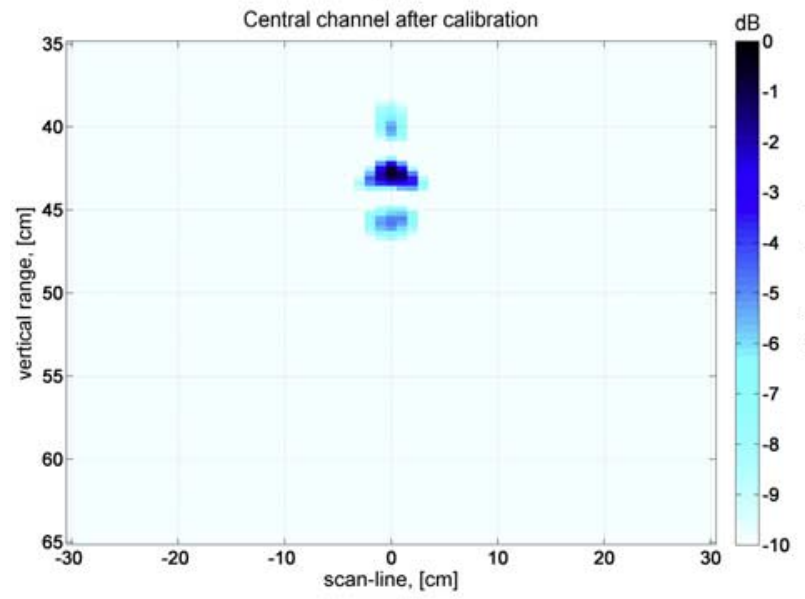

(c)

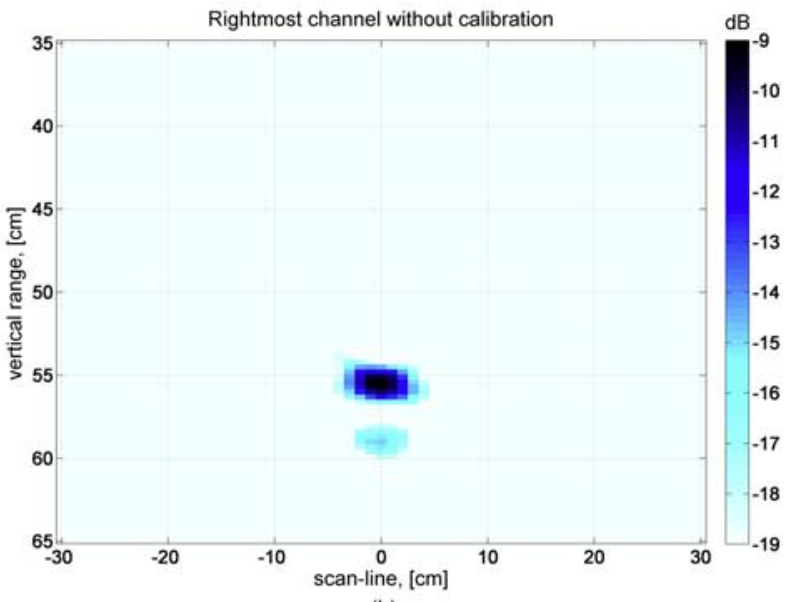

(b)

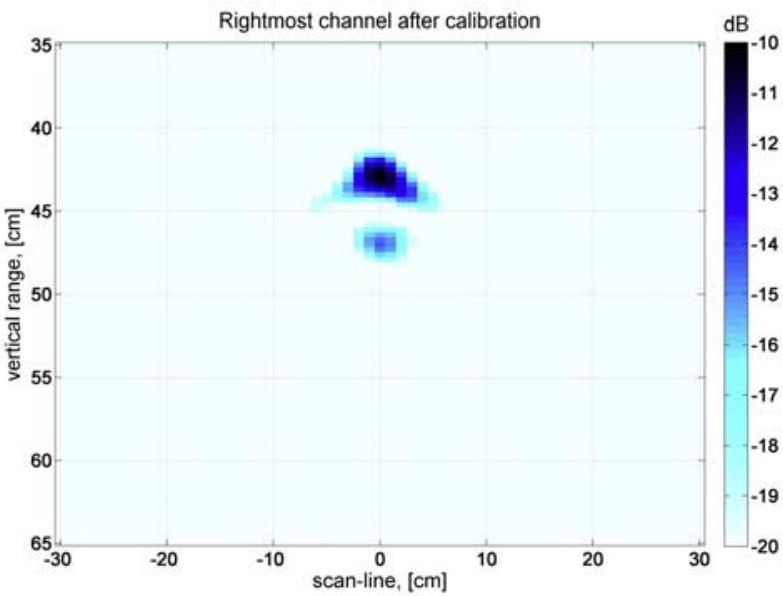

(d)

Figure 4. Array calibration: (a)-(b) migrated B-scans for the central and rightmost channels without "time zero" calibration; (c)-(d) migrated B-scans for the central and rightmost channels after "time zero" calibration.

channel, which is caused by the footprint of Tx-antenna (Figure 1c).

\section{Estimation of Array Footprint and Cross-Range Resolution}

[17] The complete 3-D focusing after the full calibration results in a high quality image of the metal sphere given in Figure 5a that is a horizontal slice across the target image shown with $10 \mathrm{~dB}$ dynamic range. The size of the sphere's image in the plane of array is $5 \mathrm{~cm}$ at $-10 \mathrm{~dB}$ level while in the mechanical scan direction this size becomes $6 \mathrm{~cm}$ because the synthetic aperture is smaller than the real aperture, more specifically $60 \mathrm{~cm}$ versus $84 \mathrm{~cm}$. This particular image of the sphere can be considered as an estimate of the footprint of the focused array which immediately gives an idea of the cross-range resolution. Furthermore, the cross-section of the footprint in the plane of array represents an array pattern with the main lobe and sidelobes in the near field. A vertical slice taken across the 3-D image in the plane of array is shown in Figure $5 \mathrm{~b}$ with $50 \mathrm{~dB}$ dynamic range. It illustrates that the amplitude correction narrows the main lobe but it increases the level of sidelobes by $6 \mathrm{~dB}$ at most. This can be understood in terms of tapering functions widely used in digital beamforming when the array pattern is interrelated with the amplitude distribution of the received signal over array via a Fourier transform [Farina, 1992]. Smooth tapering functions like the one shown in Figure 1d reduce sidelobes of the array pattern by the price of a wider main lobe. Our amplitude correction makes it vice versa which improves the resolution. The 


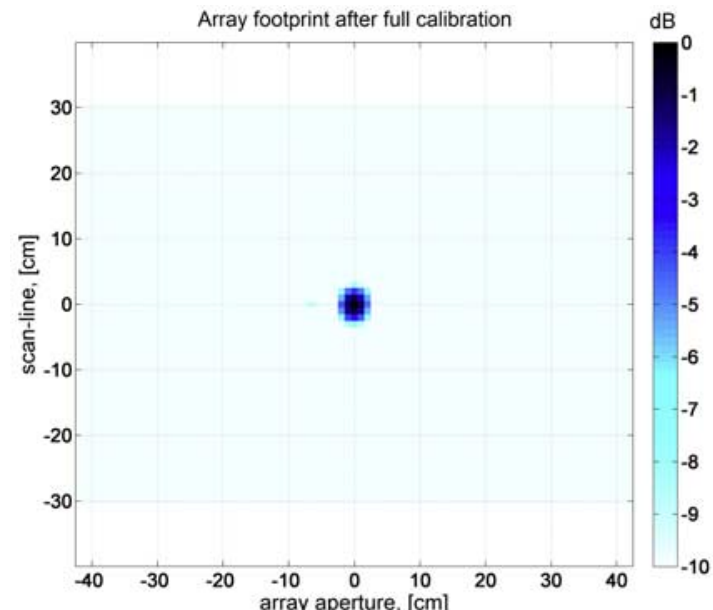

(a)

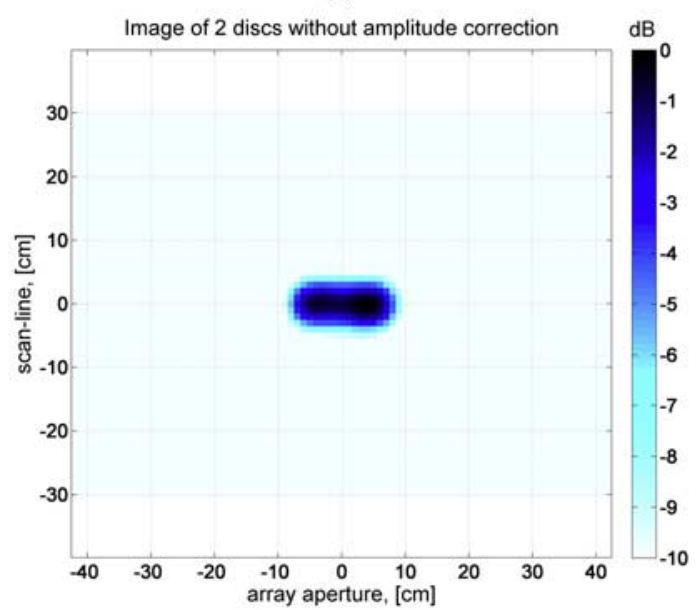

(c)

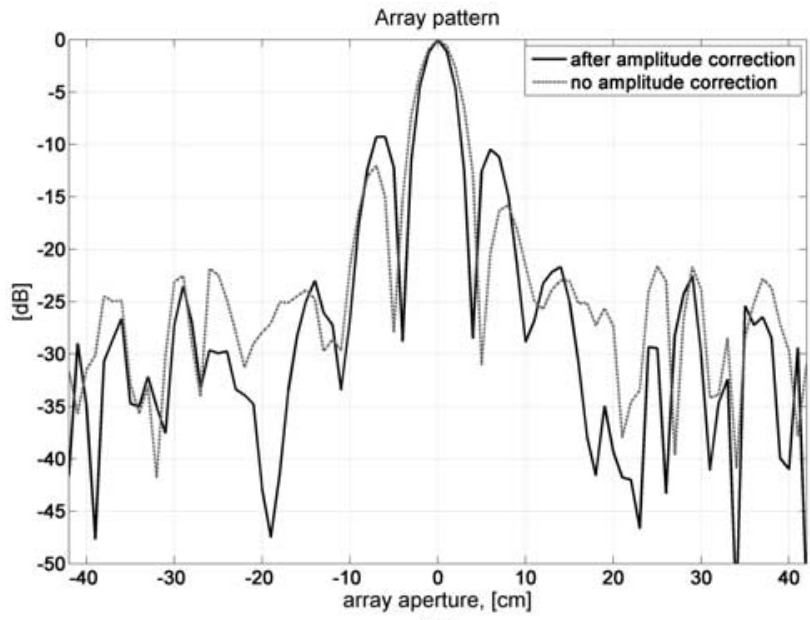

(b)

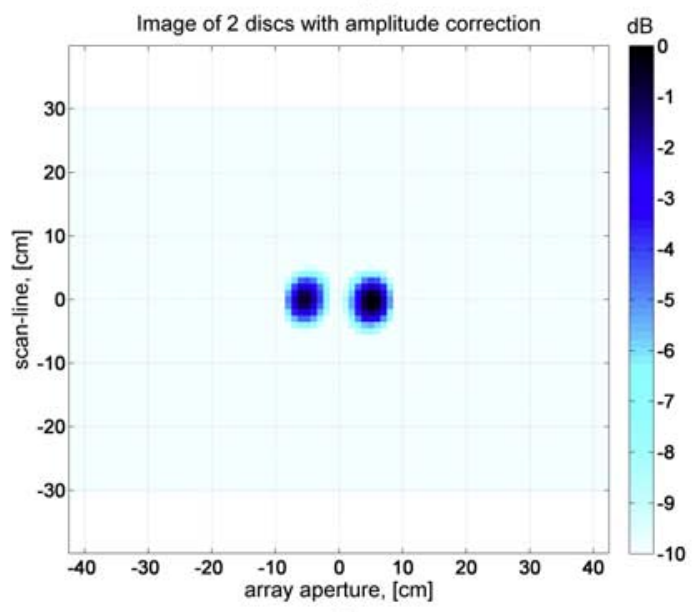

(d)

Figure 5. Array footprint and cross-range resolution: (a) horizontal slice of 3-D image across a small metal sphere as a footprint of focused array with full calibration; (b) cross-section of the footprint in the plane of array; (c) image of two metal discs of $5 \mathrm{~cm}$ diameter, separated by $4 \mathrm{~cm}$, without amplitude correction; (d) image of two metal discs obtained with amplitude correction.

maximal level of sidelobes increases to $-9 \mathrm{~dB}$ that does not bring much noise to the reconstructed image of the small scatterer as shown in Figure 5a.

[18] In order to estimate the cross-resolution of the array directly, an experiment with two small metal discs (of $5 \mathrm{~cm}$ diameter) has been carried out. The discs were placed on the array line symmetrically with respect to Tx-antenna, and a number of scans were performed for different separations between the discs. Due to the amplitude correction the discs can be resolved already at $4 \mathrm{~cm}$ separation between their edges, which is demonstrated in Figures $5 \mathrm{c}$ and $5 \mathrm{~d}$. This result fits the estimated array footprint very well, and the small difference can be explained by different size of the sphere and the discs. Note that the size of target image depends on the used threshold that is in our case $-10 \mathrm{~dB}$ level from the peak value of focused signal.

\section{Subsurface Imaging of Landmines}

[19] To test the capability of the developed focusing technique to image buried landmines subsurface measurements have been carried out at TNO-DSS premises. The array was fixed on an $\mathrm{X}-\mathrm{Y}$ translation table in such a way that the height of $\mathrm{Tx}$-antenna and $\mathrm{Rx}$-array equalled to $52 \mathrm{~cm}$ and $26 \mathrm{~cm}$ respectively. The soil was dry sand with a relative dielectric constant of 3 that was measured with a time-domain reflectometer. The 


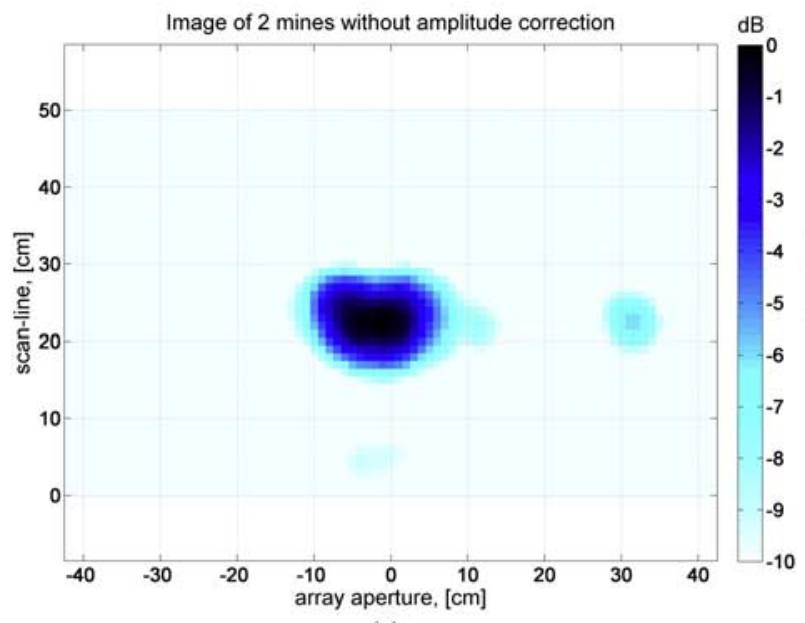

(a)

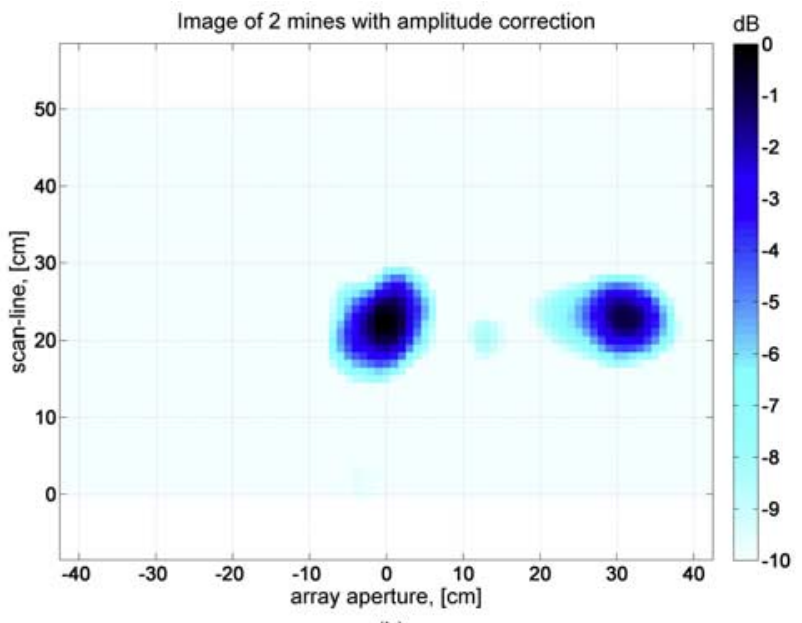

(b)

Figure 6. Imaging of buried landmines: (a) image of two PMN-2 landmines without amplitude correction; (b) image of two PMN-2 obtained with amplitude correction.

surface was flattened to decrease its impact on the array focusing at this stage of research. In case of rough surface, the clutter removal becomes more difficult while the clutter residues result in artefacts in the subsurface image. Moreover, the distance between Rx-antenna and the ground varies within the array aperture, which leads to errors in estimation of the travel times and then to degradation of the image. However, those factors are independent of the optimal weighting function and, as such, they should be considered separately.

[20] The selected targets were two identical mock landmines PMN-2 with a $13 \mathrm{~cm}$ diameter and $5.5 \mathrm{~cm}$ height. The dielectric constant of PMN-2 is of about 2.8, and the quantity of metal is $1 \mathrm{~g}$. The landmines were buried at a depth of $5 \mathrm{~cm}$ in such a way that one landmine was placed under the array centre while the other was buried close to the array right edge.

[21] The acquired data set was preprocessed as described above and then focused with algorithm (1). For the subsurface imaging a two-medium model was used with an accurate solution of the fourth-order equation for a refraction point. The imaging of one horizontal slice $50 \times 84 \mathrm{~cm}$ with a $1 \times 1 \mathrm{~cm}$ cell takes $340 \mathrm{~s}$ in $\mathrm{MATLAB}^{\mathrm{TM}}$ v. 7 on a desktop PC with $3 \mathrm{GHz}$ Pentium-4 processor and 512 MB RAM.

[22] Images obtained by focusing without and with the amplitude correction are shown respectively in Figures 6a and $6 \mathrm{~b}$ as horizontal slices across the landmines. The amplitude correction results in a better focusing and gives similar images of the identical targets independently of their position within the array aperture. However, one can notice in Figure $6 \mathrm{~b}$ a slight artefact between the two images of landmines, which is caused by the increased level of their sidelobes as illustrated in Figure 5b. This is a relatively small price for the possibility not only to improve the cross-range resolution but, what is even more important, to detect a target at the edge of array.

\section{Discussion and Conclusion}

[23] IRCTR developed a novel vehicular array-based GPR for humanitarian demining that comprises a single transmit antenna and linear receive array acquiring the scattered field by all its channels simultaneously. Due to directional properties of the transmit antenna the amplitude distribution of the received signals over the array aperture is nonuniform which degrades the cross-range resolution and leads to poor detectability of landmines buried close to the edges of array. For classical narrowband arrays and far-field operation it is well known that a uniform illumination of the array results in a sharp main lobe but high sidelobes of the array pattern while varying the illumination with a weighting function decreases the sidelobes by the price of a wider main lobe. The article considers this behavior for time-domain focusing in near field for a UWB array.

[24] The developed array technique combines a SAR procedure in the direction of vehicle's movement with a weighted focusing procedure of the array aperture in the orthogonal direction. Both procedures are based on a diffraction stacking migration algorithm that works with travel times of the radar signal. The weight coefficients for the array channels are proposed to estimate as inversed peak values of the antenna coupling between transmit and receive antennas. The measurement of these amplitude correction parameters along with "time zero" 
correction parameters constitutes a calibration of the array channels that can be performed on a surface laid small metal sphere.

[25] It has been demonstrated by experiment that the proposed amplitude correction improves the footprint of the focused array from $7 \mathrm{~cm}$ to $5 \mathrm{~cm}$ at $-10 \mathrm{~dB}$ level of image intensity by the price of increased sidelobes (the peak of the first sidelobe is increased from $-12 \mathrm{~dB}$ to $-9 \mathrm{~dB})$. The cross-range resolution, which is interrelated with the footprint, was measured and evaluated separately with a similar result of $4 \mathrm{~cm}$ at $-10 \mathrm{~dB}$ level. The capability of the proposed focusing technique to image buried antipersonnel landmines has been proven by subsurface measurements. The amplitude correction gives a proper image of a typical landmine independently of its position within the array aperture by a price of small artefacts due to increased sidelobes. In general, it has been shown that specific aspects of array radar imaging can be enhanced by applying a weighting function to the array channels in UWB case under near-field conditions.

[26] Acknowledgments. The authors cordially thank and acknowledge Pascal Aubry, IRCTR engineer, and Jan Rhebergen, TNO fellow, for their helpful, effective support during the measurements. Development of the array-based GPR has been supported by the Dutch Ministry of Defense in the framework of the contract DMO/HDM/DR\&D N01/26-10.

\section{References}

Chignell, R. J., and M. Hatef (2004), LOTUS - A real time integrated sensor suite for anti-personnel mine detection, incorporating the MINEREC GPR, paper presented at Xth International Conference on Ground Penetrating Radar, Delft, Netherlands, 21-24 June.

Daniels, D. J. (2004), Ground Penetrating Radar, 2nd ed., Inst. of Electr. Eng., London.

DEMINE partners (2002), Public report D16, Eur. Proj. 29902 DEMINE. (Available at http://www.eudem.vub.ac.be/files/ demine_final_report.pdf)

Duchesne, L., L. Durand, and P. Garreau (2003), Development of a compact MS-GPR radar based on multi-sensors technology, paper presented at 2nd International Workshop on Advanced Ground Penetrating Radar, Delft, Netherlands, 14-16 May.

Farina, A. (1992), Antenna-Based Signal Processing Techniques for Radar Systems, pp. 19-25, Artech House, Boston.
Geo-Centers EFGPR (2000), web-site. (Available at http:// www.geo-centers.com/products/EFGPR-Overview-1-03.pdf)

Gilmore, C., H. Su, I. Jeffrey, M. Phelan, and J. LoVetri (2007), Comparison of seismic migration and stripmap SAR imaging methods for GPR for landmine detection, in UltraWideband Short Pulse Electromagnetics, vol. 7, edited by F. Sabbat, E. Mokole, U. Schenk, and D. Nitsch, pp. 646654, Springer, New York.

Groenenboom, J., and A. G. Yarovoy (2002), Data processing and imaging in GPR system dedicated for landmine detection, Subsurf. Sens. Technol. Appl., 3(4), 387-402.

$\mathrm{Gu}$, K., G. Wand, and J. Li (2005), Migration based SAR imaging for ground penetrating radar systems, IEE Proc. Radar Sonar Navig., 151(5), 317-325.

Johansson, E. M., and J. E. Mast (1994), Three-dimensional ground penetrating radar imaging using synthetic aperture time-domain focusing, Proc. SPIE, 2275, 205-214.

Sato, M., Y. Hamada, X. Feng, F. N. Kong, Z. Zeng, and G. Fang (2004), GPR using an array antenna for landmine detection, Near Surf. Geophys., 2, 3-9.

Taylor, J. D. (2000), Ultra-Wideband Radar Technology, CRC, New York.

U.S. Humanitarian Demining R\&D Program (2007), web-site. (Available at http://www.humanitarian-demining.org/dettech. asp)

Warhus, J. P., J. M. Hernandez, S. D. Nelson, E. M. Johansson, and H. Lee (1993), Ground penetrating, imaging radar for bridge inspection, in Engineering Research, Development, and Technology, UCRL-53868-92, Lawrence Livermore Natl. Lab., Livermore, Calif., March.

Yarovoy, A. G., T. G. Savelyev, P. J. Aubry, P. E. Lys, and L. P. Ligthart (2006), UWB array-based sensor for near-field imaging, IEEE Trans. MTT, 55, 1288-1295.

Zhuge, X., T. G. Savelyev, A. G. Yarovoy, and L. P. Ligthart (2007), Subsurface imaging with UWB linear array: evaluation of antenna step and array aperture, paper presented at International Conference on Ultra-Wideband, Singapore, 24-26 Sept.

L. P. Ligthart, T. G. Savelyev, and A. G. Yarovoy, International Research Centre for Telecommunications and Radar, Delft University of Technology, Mekelweg 4, 2628 CD, Delft, Netherlands. (1.p.ligthart@irctr.tudelft.nl; t.g.savelyev@ irctr.tudelft.nl; a.yarovoy@irctr.tudelft.nl) 